\title{
Autophagy relieves the function inhibition and apoptosis-promoting effects on osteoblast induced by glucocorticoid
}

\author{
YUDI HAN ${ }^{1}$, LIHAI ZHANG ${ }^{1}$, YALING XING ${ }^{2}$, LICHENG ZHANG ${ }^{1}$, \\ XIAOJUAN CHEN ${ }^{2}$, PEIFU TANG ${ }^{1}$ and ZHONGBIN CHEN ${ }^{2}$ \\ ${ }^{1}$ Department of Orthopaedics, Chinese People's Liberation Army General Hospital, Beijing 100853; \\ ${ }^{2}$ Division of Infection and Immunity, Department of Electromagnetic and Laser Biology, \\ Beijing Institute of Radiation Medicine, Beijing 100850, P.R. China
}

Received October 26, 2016; Accepted October 20, 2017

DOI: $10.3892 /$ ijmm.2017.3270

\begin{abstract}
Autophagy may be a major mechanism by which osteoblasts (OBs) protect against the negative effects of chronic glucocorticoid (GC) usage. OBs are closely associated with the remodeling that occurs in GC-induced osteoporosis (GIO). In osteocytes, in response to stress induced by GCs, several pathways are activated, including cell necrosis, apoptosis and autophagy. However, the role of autophagy in OBs following treatment with excess GCs has not been addressed. In the current study, confocal microscopy observation of green fluorescent protein-microtubule-associated protein 1 light chain $3 \beta$ (LC3) punctuate, and western blotting for LC3II and Beclin 1 were performed for detection of autophagy in the MC3T3-E1 osteoblastic cell line. Flow cytometry and western blotting were used for the examination of apoptosis and expression of BAX apoptosis regulator (Bax)/apoptosis regulator $\mathrm{Bcl}-2$ (Bcl-2). The expression of genes associated with osteoblastic function, runt-related transcription factor $2, \alpha-1$ type 1 collagen and osteocalcin, were measured by reverse transcription-quantitative polymerase chain reaction. The results indicated that autophagy was induced in OBs during dexamethasone (Dex) treatment in a dose-dependent manner. The level of autophagy did not continue to increase over time, but peaked at $48 \mathrm{~h}$ and then decreased gradually. Subsequently, flow cytometry was used to demonstrate that inhibition of autophagy induced apoptosis in OBs under Dex treatment, and was associated with the
\end{abstract}

Correspondence to: Dr Peifu Tang, Department of Orthopaedics, Chinese People's Liberation Army General Hospital, 28 Fuxing Road, Beijing 100853, P.R. China

E-mail: pftang301@163.com

Dr Zhongbin Chen, Division of Infection and Immunity, Department of Electromagnetic and Laser Biology, Beijing Institute of Radiation Medicine, 27 Taiping Road, Beijing 100850, P.R. China

E-mail: chenzb@bmi.ac.cn

Key words: osteoblast, autophagy, apoptosis, glucocorticoids, osteoporosis upregulation of Bax and the downregulation of $\mathrm{Bcl}-2$ protein expression. Furthermore, the data suggested that the inhibition of autophagy also suppressed the expression of osteoblastic genes. By contrast, the stimulation of autophagy maintained the gene expression level under Dex treatment. The data revealed that autophagy is an important regulator of osteoblastic apoptosis through its interaction with $\mathrm{Bax} / \mathrm{Bcl}-2$, and maintains the osteoblastic function of MC3T3-E1 cells following GC exposure. In addition, these results indicated that the suppression of autophagy in OBs under chronic GC therapy may increase the prevalence of GIO and fragility fractures.

\section{Introduction}

Chronic glucocorticoid (GC) therapy is widely used in clinical medicine as an effective way in treating skin disease, inflammatory/autoimmune diseases, including rheumatoid arthritis and chronic obstructive pulmonary disease, and preventing organ transplantation rejection (1). However, long-term use of GCs causes various side-effects, and the most common one being GC-induced osteoporosis (GIO) (2). Approximately half of patients with rheumatoid arthritis receiving chronic GCs therapy experience an osteoporotic fracture; base-line data from randomized clinical trials reported the prevalence of vertebral fractures is $\sim 30 \%$ (3). It was also reported that long-term intake of GCs leads to bone infarction and osteonecrosis of the femoral head, causing a significant public health issue (4). Bone loss in GIO is a biphasic process, bone resorption significantly increases within the first 3 months, peaks at 6 months, followed by a slower annual loss of $\sim 3 \%$ for the duration of GC administration (5). This is caused by the accelerated lifespan of osteoclasts in the early phase of bone resorption. However, in the long term, osteoclastic activity is usually inhibited by GCs (6). By contrast, osteoblasts (OBs) are directly and consistently modulated by GCs, whereby the production of new OBs precursors is decreased and the mature, matrix-secreting OBs experience premature apoptosis. Therefore, although both bone resorption and formation are suppressed by chronic GCs therapy, bone formation is suppressed more than bone resorption (7-9). 
These histological characteristics are quite different from those in other forms of osteoporosis in which osteoclasts have an important role. Thus, more attention should be paid to OBs when investigating GIO.

Autophagy is a lysosomal degradation pathway that is essential for cell growth, survival, differentiation, development and homeostasis. It is a highly regulated process that mainly involves the formation of autophagosomes, which will fuse with the lysosomes and form autolysosomes. Subsequently, degradation occurs and the amino acids or other small molecules are delivered to the cytoplasm for energy production or recycling (10). GC therapy leads to accumulation of damaged proteins, oxygen radicals and degraded nucleic acids (11). Autophagy is the only intracellular degradative mechanism known to remove dysfunctional organelles and/or oxidized proteins, and it is also activated as an attempt to survive under stress $(12,13)$. Despite the ability in which autophagy acts as a survival strategy to preserve cell viability, it may also lead to a programmed cell death process, because of the excessive activation of this self-degrading system (14). Therefore, autophagy has been reported as a 'double-edged sword' (15). It was previously reported that autophagy was induced prior to apoptosis during lymphoid cell death caused by dexamethasone (Dex) (16). Another study reported that GC treatment could induce autophagy in mouse osteocytes. Inhibition of autophagy led to augmentation of the effects of GCs on osteocyte viability (11). The autophagic process is also induced in OBs during mineralization, and autophagy deficiency reduced mineralization capacity, which was proved by knockdown of autophagy-essential genes and OB-specific autophagy-deficient mice (17).

However, whether autophagy is involved in the effect of GCs on OBs is unknown. We hypothesize that autophagy is one of the mechanisms by which OBs respond to GC treatment. In this study, it was investigated whether GC treatment of OBs induced autophagy. In addition, as the association between autophagy and apoptosis was unclear, as previously described, whether suppression of autophagy altered the negative effects of exogenous GCs on OBs was also examined. The present observations may offer a novel finding that autophagy is a major mechanism by which OBs protect themselves against the detrimental effect of GCs.

\section{Materials and methods}

Reagents and antibodies. The enhanced green fluorescent protein-microtubule-associated protein 1 light chain $3 \beta$ (eGFP-LC3B) plasmid was provided by Dr Shaobo Xiao (Huazhong Agricultural University, Wuhan, China) (18). The antibodies used were: anti-microtubule-associated protein 1 light chain 3 3 (anti-LC3B; cat. no.L7543; Sigma-Aldrich; Merck KGaA, Darmstadt, Germany); anti-Beclin 1 (cat. no. PD017; MBL International Co., Woburn, MA, USA); anti-BAX apoptosis regulator (Bax; cat. no. 50599-2-Ig; Proteintech, Chicago, IL, USA); anti-apoptosis regulator Bcl-2 (Bcl-2; cat. no. PRS3335; Sigma-Aldrich; Merck KGaA); anti- $\beta$-actin (cat. no. AA128; Beyotime Institute of Biotechnology, Haimen, China). Dex (cat. no. D4902), 3-methyladenine (3-MA; cat. no. M9281) and rapamycin (cat. no. R8781) were all obtained from Sigma-Aldrich (Merck KGaA). Dex, rapamycin and 3-MA were all dissolved in dimethyl sulf- oxide (cat. no. D4540; Sigma-Aldrich; Merck KGaA). The stock solution concentrations were $500 \mu \mathrm{mol} / \mathrm{l}$ for rapamycin, $100 \mathrm{mmol} / \mathrm{l}$ for 3 -MA. The working solution concentrations were $500 \mathrm{nmol} / \mathrm{l}$ for rapamycin and $400 \mu \mathrm{mol} / \mathrm{l}$ for 3 -MA.

Cell culture. The MC3T3-E1 cell line was obtained from the Cell Resource Center, Peking Union Medical College (Beijing, China; the headquarters of National Infrastructure of Cell Line Resource). The cell line was confirmed to have no mycoplasma contamination by polymerase chain reaction (PCR). Its species origin was confirmed by PCR. The identity of the cell line was authenticated with Short Tandem Repeat profiling (CODIS; FBI). All the results can be viewed at www.cellresource.cn. Cells were cultured in $\alpha$-minimum essential medium (cat. no. 12571-063) with $10 \%$ (v/v) fetal calf serum (cat. no. 16140-071; both from Gibco; Thermo Fisher Scientific, Inc., Waltham, MA, USA), and antibiotics (100 IU/ml penicillin $\mathrm{G}$ and $100 \mathrm{Ag} / \mathrm{ml}$ streptomycin) in a humidified incubator with $5 \% \mathrm{CO}_{2}$ at $37^{\circ} \mathrm{C}$. A total of $5 \times 10^{5}$ cells/well were cultured in 6 -well plates and then exposed to Dex of various doses at $10^{-8}, 10^{-6}$ and $10^{-4} \mathrm{~mol} / 1$ for 24, 48, 72 and $96 \mathrm{~h}$. Autophagy inhibitor 3-MA was added to cells $1.5 \mathrm{~h}$ before incubated with Dex as negative control group. Autophagy agonist rapamycin was added $6 \mathrm{~h}$ ahead of each time-point as positive control group. Culture medium was replaced with the same concentration GC at interval of $48 \mathrm{~h}$.

Confocal microscopy. MC3T3-E1 cells were cultured on glass coverslips in 35-mm plates. For the detection of autophagosomes, eGFP-LC3B plasmid ( $2.5 \mu \mathrm{g} /$ well) was transfected into $1 \times 10^{5}$ cells/well using Lipofectamine ${ }^{\circledR}$ LTX and Plus reagent (cat. no. 15338-100; Invitrogen; Thermo Fisher Scientific, Inc.) according to manufacturer's instructions. Cells were then treated with $10^{-8}, 10^{-6}$ and $10^{-4} \mathrm{~mol} / \mathrm{l}$ Dex in complete culture medium for 24, 48, 72 and $96 \mathrm{~h}$. Rapamycin $(500 \mathrm{nmol} / \mathrm{l})$ was added $6 \mathrm{~h}$ before each time-point as positive control, and $250 \mu \mathrm{mol} / 13-\mathrm{MA}$ was added $1.5 \mathrm{~h}$ before Dex as negative control. Following fixation with phosphate-buffered saline (PBS) containing $4 \%(\mathrm{w} / \mathrm{v})$ paraformaldehyde at $37^{\circ} \mathrm{C}$ for $30 \mathrm{~min}$ and staining with $0.1 \mu \mathrm{g} / \mathrm{ml}$ DAPI at $37^{\circ} \mathrm{C}$ for $1 \mathrm{~min}$, glass coverslips were observed under a Zeiss LSM 510 confocal fluorescence microscope (Carl Zeiss AG, Oberkochen, Germany). Cells containing three or more GFP-LC3 puncta were defined as autophagy-positive. The percentage of cells with characteristic punctate GFP-LC3 staining relative to all GFP-positive cells was calculated as previously described (19). Three random fields were counted, and three independent experiments were performed. Analysis of images was performed using ImageJ (National Institutes of Health, Bethesda, MD, USA).

Western blot analysis. MC3T3-E1 cells were seeded into 6-well plates and incubated at $37^{\circ} \mathrm{C}$ for $18 \mathrm{~h}$. Cells were subsequently treated with Dex at different concentrations for 24-96 $\mathrm{h}$ as previously described. At each time-point, cells were washed with PBS twice and incubated in a cold buffer containing $0.5 \%$ Triton X-100, $150 \mathrm{mmol} / \mathrm{l} \mathrm{NaCl}, 12.5 \mathrm{mmol} / \mathrm{l}$ $\beta$-glycerolphosphate, $1.5 \mathrm{mmol} / 1 \mathrm{MgCl}_{2}, 2 \mathrm{mmol} / 1$ EDTA, $10 \mathrm{mmol} / \mathrm{l} \mathrm{NaF}, 1 \mathrm{mmol} / 1 \mathrm{Na}_{3} \mathrm{VO}_{4}, 2 \mathrm{mmol} / \mathrm{l}$ dithiothreitol and protease inhibitor cocktail (cat. no. P8340; Sigma-Aldrich). Centrifugation was performed to obtain cell extracts at 
$10,000 \times \mathrm{g}$ at $4^{\circ} \mathrm{C}$ for $10 \mathrm{~min}$, and protein concentration was determined using BCA Protein Assay kit (cat. no. pp0101; Biomed, Beijing, China). Protein samples were mixed with $30 \mu 12 X$ SDS-PAGE sample buffer, boiled for $10 \mathrm{~min}$, separated on $12 \%$ SDS-PAGE gel (30 $\mu \mathrm{g} / \mathrm{well})$, and electrotransferred onto a polyvinylidene difluoride membrane. Blots were blocked for $1 \mathrm{~h}$ with Tris-buffered saline $(50 \mathrm{mmol} / \mathrm{l}$ Tris-HCl, pH 7.4, 150 mmol/1 NaCl)-0.05\% Tween-20 (TBST) supplemented with $5 \%$ non-fat milk at room temperature and incubated overnight at $4^{\circ} \mathrm{C}$ with primary antibody [Bax (dilution, 1:1,000), Bcl-2 (dilution, 1:1,000), Beclin 1 (dilution, 1:1,000), LC3 (dilution, 1:1,500) and $\beta$-actin (dilution, $1: 2,000)]$. Membranes were than washed three times in 1X TBST buffer, and subsequently incubated with horseradish peroxidase-conjugated secondary antibodies at room temperature (cat. nos. ZF0136 and ZF0312; OriGene Technologies, Inc., Beijing, China) for $45 \mathrm{~min}$ and washed again prior to detection of signal with Western Lighting Plus-ECL reagents (cat. no. P1010; Applygen Technologies, Inc., Beijing, China). Densitometry analysis was performed using ImageJ $2 \mathrm{x}$ (National Institutes of Health, Bethesda, MD, USA).

Measurement of apoptosis by flow cytometry. MC3T3-E1 cells $\left(1 \times 10^{6}\right.$ per well) were plated in a 6 -well plate at $37^{\circ} \mathrm{C}$. Following treatment with Dex, 3-MA and rapamycin, apoptosis incidence in OBs was detected by using the Annexin V-fluorescein isothiocyanate (FITC) staining kit (BD Pharmingen, Franklin Lakes, NJ, USA). Propidium iodide (PI) staining also was performed at the same time using the same kit (20). Apoptotic cells, including those staining positive for Annexin V-FITC and negative for PI and those were double positive, were counted and represented as a percentage of the total cell count. Flow cytometry analysis was performed by BD FACSDiva software (v 6.1.3; BD Biosciences, San Jose, CA, USA).

Gene expression analysis. Total RNA was isolated using TRIzol reagent (Thermo Fisher Scientific, Inc.) according to the manufacturer's instructions. Total RNAs $(1 \mu \mathrm{g})$ were then reverse-transcribed using RayScript cDNA synthesis kit (GK8030; Generay Biotech Co., Ltd., Shanghai, China), cDNAs were subjected to reverse transcription-quantitative polymerase chain reaction (RT-qPCR) analysis in an ABI PRISM 7500 system (Applied Biosystems; Thermo Fisher Scientific, Inc.). Reactions were performed in a $20 \mu \mathrm{l}$ final volume using $8 \mu \mathrm{l}$ diluted cDNAs and AceQ ${ }^{\mathrm{TM}} \mathrm{qPCR}$ Probe Master Mix (Q112-02; Vazyme Biotech Co., Ltd., Nanjing, China). Amplification conditions were: $95^{\circ} \mathrm{C}$ for $5 \mathrm{~min}$, followed by 40 cycles of $95^{\circ} \mathrm{C}$ for $10 \mathrm{sec}$ and $60^{\circ} \mathrm{C}$ for $34 \mathrm{sec}$. Primer sequences were as follows: Runt-related transcription factor 2 (Runx2), 5'-CAGCAGCAGCAACAACAG-3' (forward) and 5'-TGGTCCGCGATGATCTC-3' (reverse), ACAGCAGCAGCAGCAGCAGCA (probe); osteocalcin $(O c n)$, 5'-GAGCTTAACCCTGCTTGTG-3' (forward) and 5'-CTGCTGTGACATCCATACTTG-3' (reverse), ATC AGACCAGTATGGCTTGAAGACCGC (probe); $\alpha-1$ type 1 collagen (Collal), 5'-CTGGCAAGAATGGAGATGATG-3' (forward) and 5'-TCGGTGTCCCTTCATTCC-3' (reverse), TGTTCCAGGCAATCCACGAGCACC (probe); GAPDH, 5'-GCCTTCCGTGTTCCTACC-3' (forward) and 5'-CCTCAG TGTAGCCCAAGATG-3' (reverse), TGCCTGCTTGACCAC
CTTCTTGATGT (probe). Each gene was normalized to GAPDH by relative quantification and the cycle quantification $\left(2^{-\Delta \Delta \mathrm{Cq}}\right)$ method was used to calculate relative gene expression levels (21).

Statistical analysis. All data were expressed as the mean \pm standard deviation of at least three independent experiments. Differences among the three groups were analyzed by one-way analysis of variance followed by the least significant difference test or the Student-Newman-Keuls test using the statistics package SPSS 19.0 (IBM Corp., Armonk, NY, USA). Other comparisons were performed using the Student's t-test except for percentage comparison for which the Kruskal-Wallis test was used. $\mathrm{P}<0.05$ was considered to indicate a statistically significant difference.

\section{Results}

Dex induces autophagy in a dose-dependent manner in MC3T3-E1 cells. To determine whether GCs regulate autophagy, the impact of Dex on formation of autophagosomes in MC3T3-E1 cells was examined. MC3T3-E1 cells were transfected with an eGFP-LC3B-expressing plasmid (22). At $24 \mathrm{~h}$ post-transfection, cells were fixed, and examined for evidence of autophagosome formation by confocal fluorescence microscopy. eGFP-LC3 fluorescence microscopy is a well-accepted method for monitoring autophagosome formation (23). As presented in Fig. 1A and B, MC3T3 cells had very low basal autophagic activity, with $<2 \%$ of cells expressed punctate eGFP-LC3. However, treatment with $10^{-8}, 10^{-6}$ and $10^{-4} \mathrm{~mol} / 1$ Dex stimulated autophagy, leading to eGFP-LC3 puncta formation in $\sim 50 \%$ of cells. A clear redistribution of eGFP-LC3B punctate was observed, indicating the formation of autophagosomes (Fig. 1A). Semi-quantitative analysis as indicated that the increase in puncta-positive cells was dose-dependent, and rapamycin could increase the number of positive cells, while 3-MA effectively reduced it (Fig. 1B).

To validate this finding, western blotting was performed to examine the endogenous expression of LC3, which is a protein involved in the formation of autophagic phagophore membranes. Activation of autophagy is associated with the conversion of LC3 from the cytosolic, soluble form (LC3-I), to the membrane-bound form (LC3-II). This ubiquitin-like modification, termed lipidation, can be illustrated by a change in migration on SDS-PAGE gel and is a sensitive marker for monitoring autophagy $(23,24)$. For further validation, Beclin 1, another marker of autophagy was examined using western blotting. Beclin 1 is involved in the initial step of the autophagic process and regulates both the formation and maturation of autophagosomes $(25,26)$. Collectively, consistent with the eGFP-LC3 fluorescence data, endogenous expression of LC3II and Beclin 1 suggested that Dex increased the autophagic activity in MC3T3-E1 cells in a dose-dependent manner following treatment with Dex for $24 \mathrm{~h}$ (Fig. 1C-F).

\section{Dex-induced autophagic activity peaks at $48 h$ and decreases} over time. To understand the temporal characteristics Dex-induced autophagy, eGFP-LC3B puncta formation in MC3T3-E1 cells transfected with eGFP-LC3B was monitored in the $10^{-8}$ and $10^{-4} \mathrm{~mol} / \mathrm{l}$ Dex-treated groups over time. 


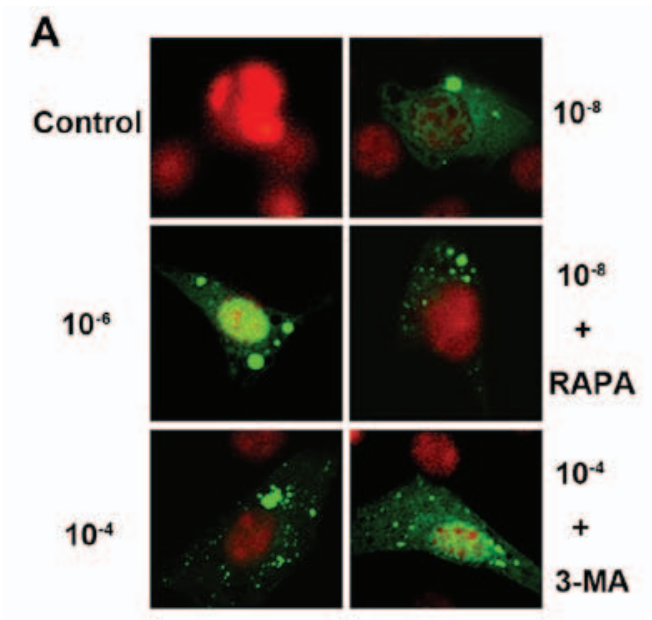

B
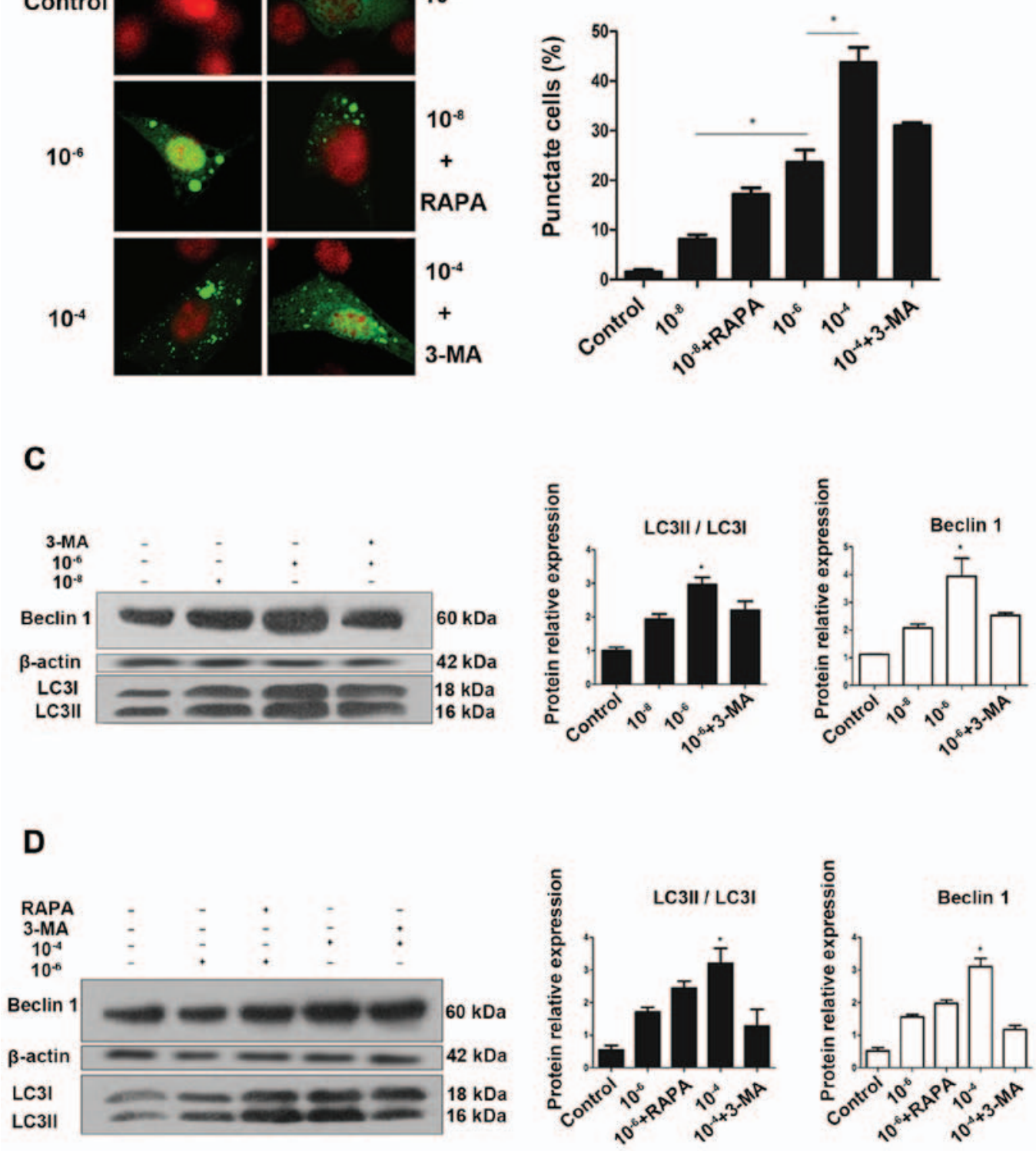

Figure 1. Autophagy is activated during glucocorticoid treatment. (A) Cells transiently transfected plasmid expressing eGFP-LC3B fusion protein, were exposed to Dex or control (no Dex) for $24 \mathrm{~h}$. Representative fluorescence microscopy images are presented (magnification, x100). (B) Control group had $<2 \%$ of cells expressing punctate eGFP-LC3. Whereas, $24 \mathrm{~h}$ treatment with $10^{-8}, 10^{-6}$ and $10^{-4} \mathrm{~mol} / 1 \mathrm{Dex}$ led to eGFP-LC3 puncta formation in $\sim 50 \%$ of cells. ${ }^{*} \mathrm{P}<0.05$ vs. control, $10^{-8}$, and $10^{-6}+3$-MA groups. Cells were exposed to culture medium containing $10^{-8}, 10^{-6}$ and $10^{-4} \mathrm{~mol} / 1 \mathrm{Dex}$ for $24 \mathrm{~h}$ with (C) 3-MA (400 $\left.\mu \mathrm{mol} / 1\right)$ added $1.5 \mathrm{~h}$ before the experiment plus (D) RAPA $(500 \mathrm{nmol} / \mathrm{l})$ added during the last $6 \mathrm{~h}$ of the experiment. Autophagy markers were evaluated by western blotting. Positive blots (right) and corresponding semiquantitative analysis (left) are presented. Values are presented as the mean \pm standard deviation ( $\mathrm{n}=3$ ). ${ }^{*} \mathrm{P}<0.05$ vs. control, $10^{-6}, 10^{-6}+\mathrm{RAPA}$, and $10^{-4}+3$-MA groups. Dex, dexamethasone; eGFP, enhanced green fluorescent protein; RAPA, rapamycin; 3-MA, 3-methyladenine; LC3, microtubule-associated protein 1 light chain $3 \beta$.

eGFP-LC3B puncta formation was observed in cytoplasm as early as $24 \mathrm{~h}$ post-transfection (Fig. 2A). In addition, eGFP-LC3B puncta increased at $48 \mathrm{~h}$ post-transfection (Fig. $2 \mathrm{~A}$ and $\mathrm{B}$ ), in accordance with an increase in LC3II protein expression levels (Fig. 2C). In the $10^{-8} \mathrm{~mol} / 1$ Dex group, $\sim 15 \%$ of cells exhibited cytoplasmic eGFP-LC3B puncta at $24 \mathrm{~h}$ post-transfection, and the number increased to $40 \%$ at $48 \mathrm{~h}$. The positive rate was $20 \%$ at $24 \mathrm{~h}$, and increased to $>50 \%$ at $48 \mathrm{~h}$ in the $10^{-4} \mathrm{~mol} / \mathrm{l}$ Dex group. However, this trend was not persistent. At $48 \mathrm{~h}$ post-transfection, in the $10^{-8}$ and $10^{-4} \mathrm{~mol} / \mathrm{l}$ groups, the percentage of cells with eGFP-LC3B puncta decreased and the eGFP-LC3B fluorescence was decreased (Fig. 2B). This was also verified by the LC3II expression levels at $48 \mathrm{~h}$ post-transfection. In accordance with these data, the endogenous Beclin 1 protein expression was upregulated in the first $48 \mathrm{~h}$, then reduced substantially afterwards (Fig. 2C and D). Rapamycin was also added to the cells at $96 \mathrm{~h}$. The results showed that the level of Beclin 1 was not significantly elevated, which suggested that autoghagy was a transient process in MC3T3-E1 cells (Fig. 2D). This result provided additional support for the feature of Dex-induced autophagic activity in MC3T3-E1 cells.

Dex induced MC3T3-E1 cell apoptosis. Following treatment with various concentrations of Dex, cell apoptosis was 
A

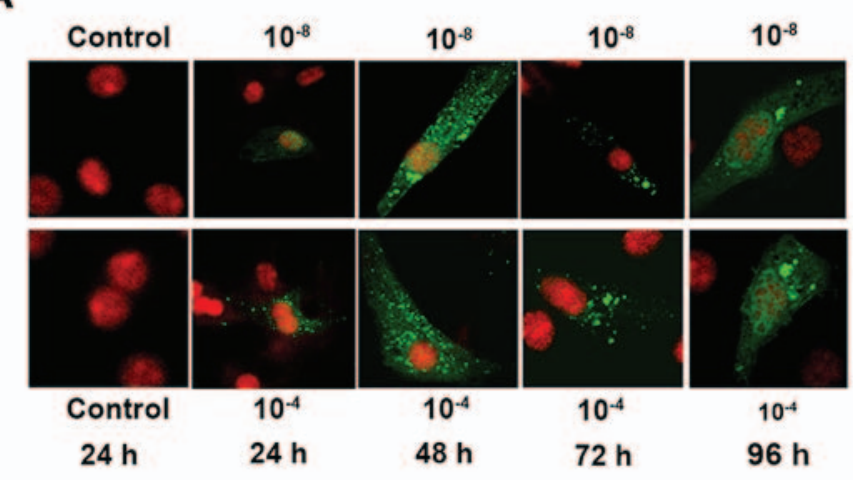

C

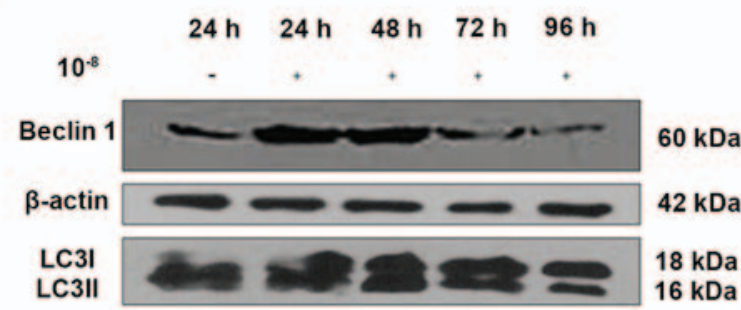

D

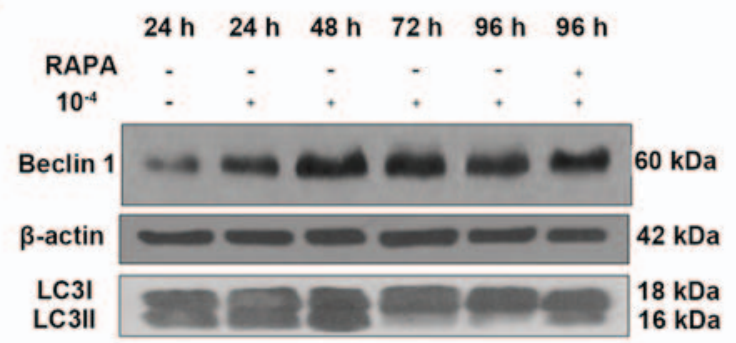

B
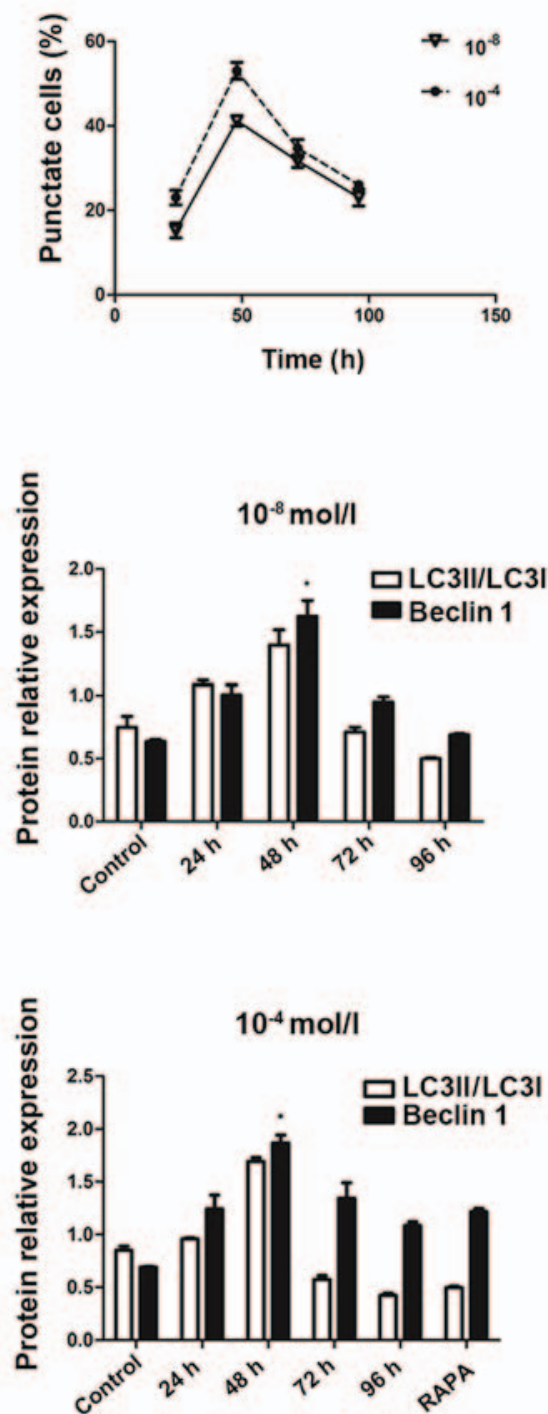

Figure 2. Generation and characterization of autophagy over time. (A) Confocal microscopy images of MC3T3-E1 cells treated with $10^{-8}$ and $10^{-4}$ mol/1 Dex for $24,48,72$ and $96 \mathrm{~h}$ (magnification, x100). (B) Broken line graph showed that enhanced green fluorescent protein-LC3 puncta positive cells reached a peak at $48 \mathrm{~h}$ and then decreased. Representative immunoblots and densitometry analysis of cells treated with (C) Dex at $10^{-8}$ or (D) Dex at $10^{-4}$ mol/1 for 24 to $96 \mathrm{~h}$ and RAPA (500 nmol/l) for $96 \mathrm{~h}$. Corresponding densitometric quantification showed that autophagy markers reached a peak at $48 \mathrm{~h}$ and decrease gradually after that. "P<0.05 vs. other time groups. Dex, dexamethasone; LC3, microtubule-associated protein 1 light chain 3 $\beta$; RAPA, rapamycin.

detected by Annexin V/PI double staining. Flow cytometry assays indicated a substantial increase in the FITC-positive population among cells treated with $10^{-6}$ and $10^{-4} \mathrm{~mol} / 1 \mathrm{Dex}$, compared to non-treated group $(\mathrm{P}<0.05)$. And the difference between $10^{-8} \mathrm{~mol} / \mathrm{l}$ and non-treated group has no statistical significance $(\mathrm{P}>0.05)$. But in general, the apoptotic population was increased in a dose-dependent manner (Fig. 3A and B). Similarly, when time was taken into account, it was demonstrated that the apoptotic population was also increased in all Dex treated groups over time, which indicates Dex exerted time-dependent effects on MC3T3-E1 cells.

Dex-induced autophagy protects MC3T3-E1 cells from undergoing apoptosis. To investigate the effect of autophagy on apoptosis, 3-MA, a potent pharmacological inhibitor of autophagy, was used to suppress Dex-induced autophagy.
By contrast, rapamycin was used to increase Dex-induced autophagy. The flow cytometry analysis demonstrated that 3-MA alone did not cause a marked increase in apoptosis and cell death (Fig. 4B), thus that pretreatment with 3-MA was able to block autophagy in MC3T3-E1 cells without significant cytotoxicity. However, 3-MA significantly induced apoptosis at $48 \mathrm{~h}$ when combined with Dex exposure $(\mathrm{P}<0.05$; Fig. $4 \mathrm{~A}$ and $\mathrm{B})$. This result indicated that suppression of autophagy by 3-MA was able to increase Dex-induced damage in MC3T3-E1 cells. To further confirm that apoptosis could be modulated by the regulation of autophagy, western blotting was performed to detect the expression of Bax and Bcl-2. As displayed in Fig. 4C and D, the expression of Bax was not decreased in the $10^{-8} \mathrm{~mol} / \mathrm{l}$ Dex groups compared with the control group $(\mathrm{P}>0.05)$. However, when co-treated with 3-MA, Bax expression was significantly upregulated compared with Dex treatment alone. By contrast, 


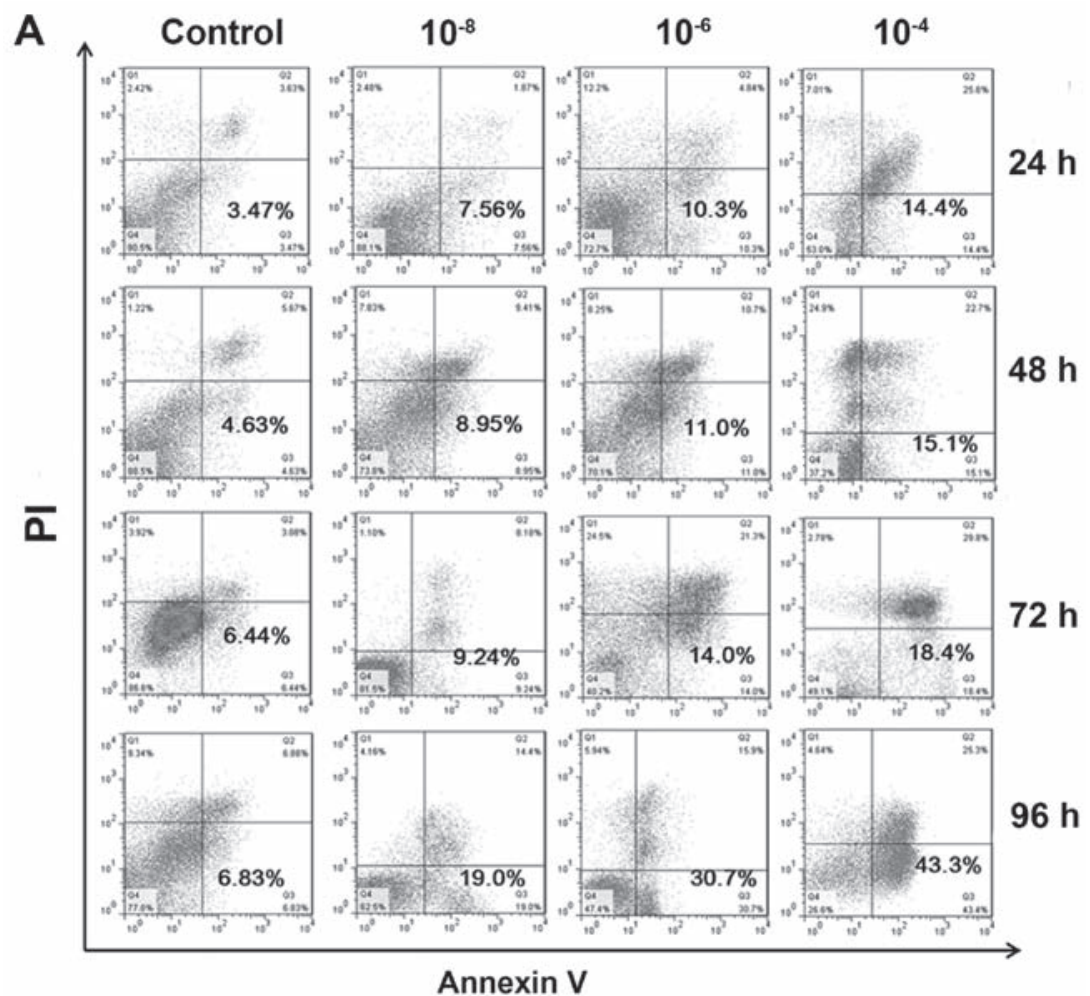

B

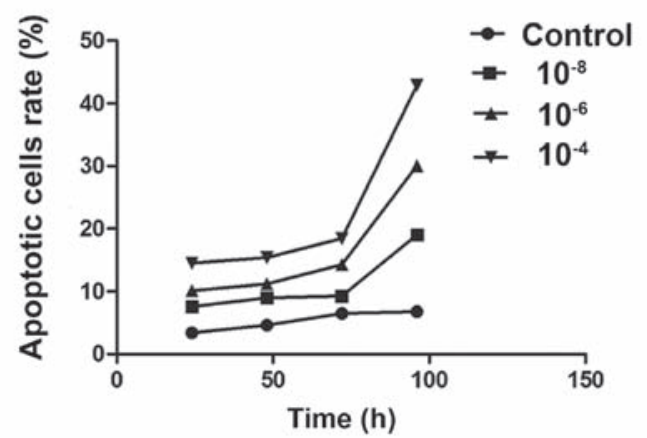

Figure 3. Apoptosis is induced during Dex treatment. (A) Annexin V-fluorescein isothiocyanate/PI staining was analyzed by flow cytometry. Representative dot plots of MC3T3-E1 cells subjected to $10^{-8}, 10^{-6}$ and $10^{-4} \mathrm{~mol} / \mathrm{l}$ Dex or control are presented with the percentage of Annexin V-positive cells indicated. (B) Population of apoptotic cells was increased in a dose-dependent manner. There was significant difference between $10^{-6}$ mol $/ 1$ and control group $(\mathrm{P}<0.05)$, $10^{-4} \mathrm{~mol} / 1$ and control group $(\mathrm{P}<0.05)$ at all time-points. Dex, dexamethasone; PI, propidium iodide.

Bax was significantly decreased compared with Dex only treatment when co-treated with rapamycin ( $\mathrm{P}<0.05$; Fig. 4C). Unlike Bax, which a pro-apoptosis protein, Bcl-2 is an anti-apoptosis protein. As shown in Fig. 4D, compared with Dex-only treatment, Bcl-2 expression was increased when cells were co-treated with rapamycin, but was reduced by co-treatment with 3-MA $(\mathrm{P}<0.05)$. These results indicated that the blockage of autophagy enhanced the apoptotic effects of Dex in MC3T3-E1 cells. Autophagy induced by Dex served a protective function, and it can be enhanced by promotion of this process.

Autophagy deficiency interferes with osteoblastic function in MC3T3-E1 cells. To further investigate whether autophagic deficiency may aggravate the inhibitory action of Dex by reducing the osteoblastic function in MC3T3-E1 cells, the expression of osteoblastic-associated genes was determined. As expected on the basis of previous findings in these osteoblastic cells (27), exposure to GCs was downregulated the expression of Runx2, Colla1 and Ocn (Fig. 5A), which are markers of bone matrix synthesis ability. Treatment of MC3T3-E1 cells with 3-MA alone did not reduce the expression of these genes $(\mathrm{P}>0.05$; Fig. 5B), and treatment with rapamycin alone did not promote their expression, thus making 3-MA and rapamycin practical tools as autophagy-inhibitor and autophagy-inducer in this study (Fig. 5B). The results demonstrated that pretreatment with 3-MA significantly reduced Runx2, Colla1 and Ocn gene expressions in cells exposed to Dex. Additionally, rapamycin promoted the expression of Ocn compared to Dex treatment (Fig. 5C).

\section{Discussion}

GCs are effective anti-inflammatory and autoimmune modulating agents, but excessive use or chronic GC therapy alters bone metabolism and produces GIO, leading to bone fragility 

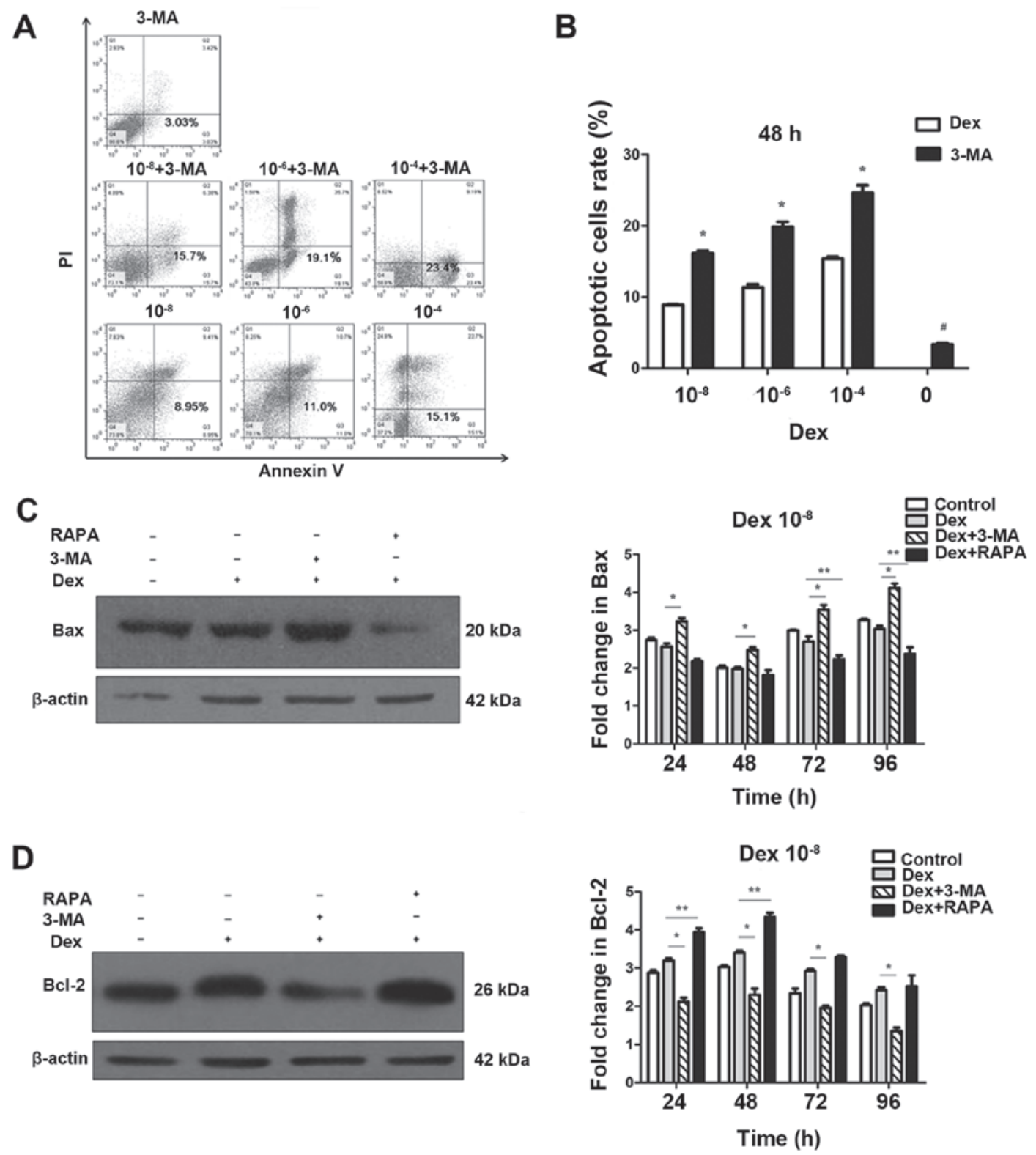

Figure 4. Regulation of autophagy interferes in the negative effects of Dex on MC3T3-E1 cells. (A) MC3T3-E1 cells were treated with Dex, in the presence or absence of chemical inhibitors of autophagy 3-MA. Annexin V-fluorescein isothiocyanate/PI staining was analyzed by flow cytometry. Representative dot plots of MC3T3-E1 cells subjected to Dex or 3-MA alone are presented. (B) Data expressed as percentage of Annexin V-positive cells (n=3). " $\mathrm{P}<0.05$, vs. corresponding control and ${ }^{\#} \mathrm{P}<0.05$ other groups vs. $3-\mathrm{MA}$ alone. Cells were treated with $10^{-8} \mathrm{~mol} / \mathrm{l}$ Dex and 3-MA (400 $\left.\mu \mathrm{mol} / \mathrm{l}\right)$ or RAPA (500 nmol/l), and the expression of (C) Bax and (D) Bcl-2 were detected by western blotting. The results were representative of three independent experiments. $\beta$-actin was used as a loading control. ${ }^{*} \mathrm{P}<0.05$; ${ }^{* *} \mathrm{P}<0.05$. 3-MA, 3-methyladenine; PI, propidium iodide; Dex, dexamethasone; RAPA, rapamycin; Bax, BAX apoptosis regulator; Bcl-2, Bcl-2 apoptosis regulator.

and bone fractures (28). Unlike senile and postmenopausal osteoporosis, in which osteolysis is activated by the increased population and function of osteoclasts, GIO is predominantly caused by the impaired function of osteogenesis (29). OBs have a central role in bone formation, and GCs directly inhibited cellular proliferation and differentiation of OB lineage cells (30), and reduce $\mathrm{OB}$ maturation and activity (31). GCs also induce $\mathrm{OB}$ and osteocyte apoptosis in vivo (32). In the current study, flow cytometry was used to demonstrated that apoptosis of MC3T3-E1 cells was induced by Dex in a dose-dependent manner. However, the mechanism of apoptosis was not clear. The expression of Bax is closely associated with mitochondrial apoptosis, which is an important factor in the regulation of cell apoptosis (33). The present study demonstrated that selective gene deletion of Bak and Bax in OBs increased their lifespan and thereby cancellous bone mass in the femur (34). Therefore, we hypothesized that Bax may be involved in the osteoblastic apoptosis caused by Dex. The current study demonstrated that Dex induced apoptosis and also induced autophagy in MC3T3-E1 cells. As shown in Fig. 1, LC3II, Beclin 1 and GFP-LC3 puncta were upregulated by Dex in a dose-dependent manner. The process of apoptosis is often accompanied by the occurrence of autophagy (35), however, the association between apoptosis and autophagy is complex (36).

Several studies have reported that inhibition of autophagy results in dysfunction and death of cells, including hemato- 

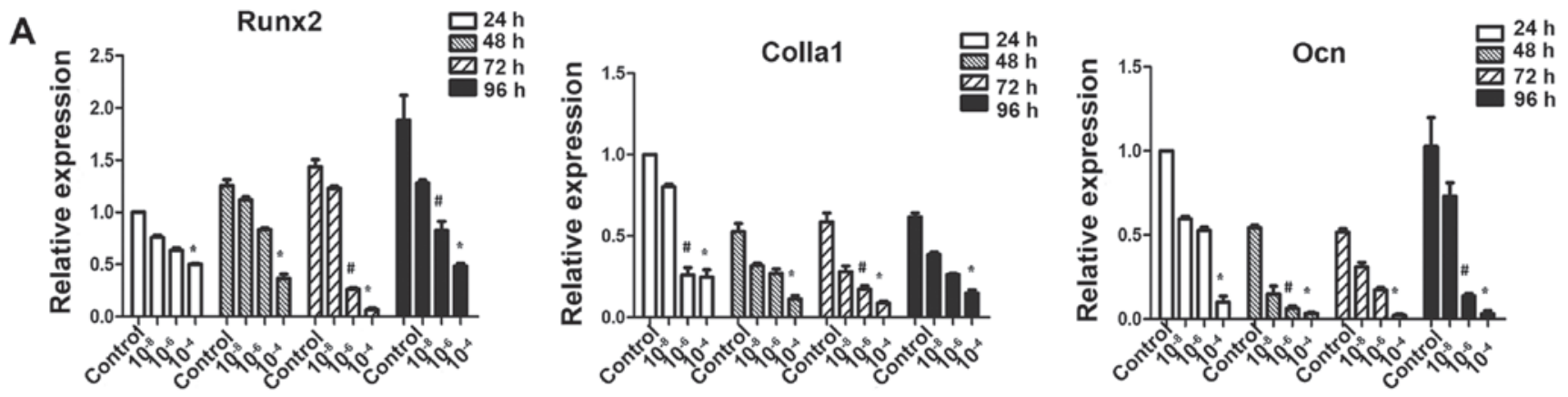

B

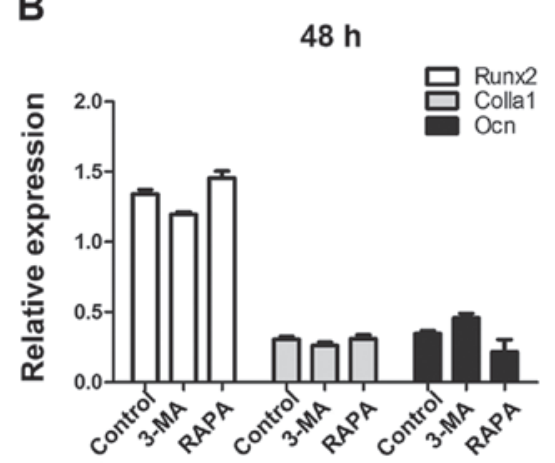

C

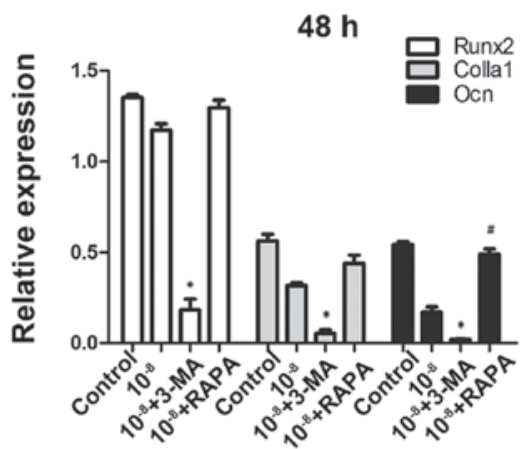

Figure 5. Autophagy has a protective effect on osteoblastic gene expressions of MC3T3-E1 cells under Dex treatment. MC3T3-E1 cells were treated with various doses of Dex. (A) Osteogenesis-associated genes Runx2, Colla1 and Ocn were examined by reverse transcription-quantitative polymerase chain reaction. " $\mathrm{P}<0.05$ vs. control; ${ }^{\prime} \mathrm{P}<0.05$ vs. control. (B) Cells treated with 3 -MA $(400 \mu \mathrm{mol} / \mathrm{l})$ or RAPA $(500 \mathrm{nmol} / \mathrm{l})$ alone were also examined, no significant difference was detected $(\mathrm{P}>0.05)$. (C) Cells were co-treated with Dex and 3-MA or RAPA. $\mathrm{P}<0.05$ vs. Dex group; "P<0.05 vs. Dex group. Dex, dexamethasone; Runx2, runt-related transcription factor 2; Colla1, $\alpha$-1 type 1 collagen; Ocn, osteocalcin; 3-MA, 3-methyladenine; RAPA, rapamycin.

poietic stem cells, pancreatic $\beta$-cells, kidney epithelium, myocardial cells and neurons (37-40). Previous studies suggested autophagy provided a mechanism for osteocytes to survive the stress after GCs exposure in vitro $(12,41)$, thus we hypothesized that suppression of autophagy in MC3T3-E1 cells may also aggravate the impact of GCs on osteoblastic function. The findings of the current study demonstrated that the suppression of autophagy by 3-MA was able to increase Dex-induced apoptosis in MC3T3-E1 cells. Furthermore, the expression of pro-apoptotic protein Bax was also upregulated following co-treatment with 3-MA and Dex, whereas it was downregulated following co-treatment with the autophagy-inducer rapamycin. To further validate the role of autophagy in mitochondrial apoptosis, the expression of Bcl-2 was examined. Bcl-2 is located in mitochondria and can stop cytochrome $c$ release into the cytoplasm, and the subsequent activation of caspase- 3 , by reducing mitochondrial membrane permeability (42). The results of the current study indicated that 3-MA significantly reduced the expression of Bcl-2 compared with the group treated with Dex only. By contrast, Bcl-2 expression was increased by co-treatment with rapamycin. These data indicated that autophagy is a pro-survival mechanism in Dex-treated MC3T3-E1 cells to reduce the incidence of apoptosis and facilitate the survival of the cells. The potential mechanism may be via regulation of $\mathrm{Bax} / \mathrm{Bcl}-2$ by autophagic process through the mitochondrial pathway. A notable finding produced by the flow cytometry analysis was that there was a marked increase in the apoptotic cell population after $72 \mathrm{~h}$ of treatment with Dex (Fig. 3), in comparison to the relative slower increase prior to $72 \mathrm{~h}$. This may be due to the decline of the activity of autophagy following the peak at $48 \mathrm{~h}$, as indicated by the confocal fluorescence microscopy data, resulting in a decreased ability to relieve the negative effect of Dex on MC3T3-E1 cells. This result suggested that autophagy may not be consistently protective if stress lasts for a prolonged time, particularly under chronic GC therapy. Therefore, this indicates that the suppression of autophagy may have an important role in the dysfunction of osteogenesis during long-term intake of GCs.

Furthermore, in accordance with the finding that autophagy facilitated the survival of cells, osteogenic gene expression as also examined by RT-qPCR in order to determine whether autophagy altered the effect of Dex on osteoblastic function. Dex reduced the gene expression of Runx2, Colla1 and Ocn, particularly at relatively higher concentrations. The expression of these genes was also determined in cells following co-treatment with 3-MA and rapamycin, with the results suggesting that osteogenic gene expression was suppressed by 3-MA and induced by rapamycin. These results indicated that autophagy not only altered the process of apoptosis, but also the function of osteoblastic cells.

In conclusion, the findings of the current study demonstrate that excess GC stimulates the process of autophagy in MC3T3-E1 cells, and inhibition of this phenomenon mediates the negative effects of Dex on cell proliferation and osteoblastic function. These results indicate that activated autophagy is a self-protective mechanism used by OBs to oppose the stress induced by excess GC. 


\section{Acknowledgements}

This study was funded by two grants from China, National High-tech R\&D Program (863 Program) (grant no. SS2012AA041604) and National Natural Science Foundation of China (grant no. 31271000).

\section{References}

1. Kanis JA, Johansson H, Oden A, Johnell O, de Laet C, Melton L JIII, Tenenhouse A, Reeve J, Silman AJ, Pols HA, et al: A meta-analysis of prior corticosteroid use and fracture risk. J Bone Miner Res 19: 893-899, 2004.

2. Van Staa TP, Laan RF, Barton IP, Cohen S, Reid DM and Cooper C: Bone density threshold and other predictors of vertebral fracture in patients receiving oral glucocorticoid therapy. Arthritis Rheum 48: 3224-3229, 2003.

3. Weinstein RS: Clinical practice. Glucocorticoid-induced bone disease. N Engl J Med 365: 62-70, 2011.

4. Mankin HJ: Nontraumatic necrosis of bone (osteonecrosis). N Engl J Med 326: 1473-1479, 1992.

5. LoCascio V, Bonucci E, Imbimbo B, Ballanti P, Adami S, Milani S, Tartarotti D and DellaRocca C: Bone loss in response to long-term glucocorticoid therapy. Bone Miner 8: 39-51, 1990.

6. Jia D, O'Brien CA, Stewart SA, Manolagas SC and Weinstein RS Glucocorticoids act directly on osteoclasts to increase their life span and reduce bone density. Endocrinology 147: 5592-5599, 2006.

7. Hong JM, Teitelbaum SL, Kim TH, Ross FP, Kim SY and Kim HJ: Calpain-6, a target molecule of glucocorticoids, regulates osteoclastic bone resorption via cytoskeletal organization and microtubule acetylation. J Bone Miner Res 26: 657-665, 2011.

8. Li H, Qian W, Weng X, Wu Z, Li H, Zhuang Q, Feng B and Bian Y: Glucocorticoid receptor and sequential P53 activation by dexamethasone mediates apoptosis and cell cycle arrest of osteoblastic MC3T3-E1 cells. PLoS One 7: e37030, 2012.

9. Naves MA, Pereira RM, Comodo AN, de Alvarenga EL, Caparbo VF and Teixeira VP: Effect of dexamethasone on human osteoblasts in culture: involvement of $\beta 1$ integrin and integrin-linked kinase. Cell Biol Int 35: 1147-1151, 2011.

10. Mizushima N: Physiological functions of autophagy. Curr Top Microbiol Immunol 335: 71-84, 2009.

11. Xia X, Kar R, Gluhak-Heinrich J, Yao W, Lane NE, Bonewald LF Biswas SK, Lo WK and Jiang JX: Glucocorticoid-induced autophagy in osteocytes. J Bone Miner Res 25: 2479-2488, 2010.

12. He C and Klionsky DJ: Regulation mechanisms and signaling pathways of autophagy. Annu Rev Genet 43: 67-93, 2009.

13. Martinet W, Agostinis P, Vanhoecke B, Dewaele M and De Meyer GR: Autophagy in disease: a double-edged sword with therapeutic potential. Clin Sci (Lond) 116: 697-712, 2009.

14. Tsujimoto Y and Shimizu S: Another way to die: autophagic programmed cell death. Cell Death Differ 12 (Suppl 2): 1528-1534, 2005.

15. Czaja MJ: Autophagy in health and disease. 2. Regulation of lipid metabolism and storage by autophagy: pathophysiological implications. Am J Physiol Cell Physiol 298: C973-C978, 2010.

16. Laane E, Tamm KP, Buentke E, Ito K, Kharaziha P, Oscarsson J, Corcoran M, Björklund AC, Hultenby K, Lundin J, et al: Cell death induced by dexamethasone in lymphoid leukemia is mediated through initiation of autophagy. Cell Death Differ 16: 1018-1029, 2009

17. Nollet M, Santucci-Darmanin S, Breuil V, Al-Sahlanee R, Cros C, Topi M, Momier D, Samson M, Pagnotta S, Cailleteau L, et al: Autophagy in osteoblasts is involved in mineralization and bone homeostasis. Autophagy 10: 1965-1977, 2014.

18. Kimura S, Noda T and Yoshimori T: Dissection of the autophagosome maturation process by a novel reporter protein, tandem fluorescent-tagged LC3. Autophagy 3: 452-460, 2007.

19. Li J, Liu Y, Wang Z, Liu K, Wang Y, Liu J, Ding H and Yuan Z: Subversion of cellular autophagy machinery by hepatitis B virus for viral envelopment. J Virol 85: 6319-6333, 2011.

20. Yang YH, Chen K, Li B, Chen JW, Zheng XF, Wang YR, Jiang SD and Jiang LS: Estradiol inhibits osteoblast apoptosis via promotion of autophagy through the ER-ERK-mTOR pathway. Apoptosis 18: 1363-1375, 2013.

21. Livak KJ and Schmittgen TD: Analysis of relative gene expression data using real-time quantitative PCR and the 2(-Delta Delta C(T)) Method. Methods 25: 402-408, 2001
22. Yang YH, Li B, Zheng XF, Chen JW, Chen K, Jiang SD and Jiang LS: Oxidative damage to osteoblasts can be alleviated by early autophagy through the endoplasmic reticulum stress pathway - implications for the treatment of osteoporosis. Free Radic Biol Med 77: 10-20, 2014.

23. Klionsky DJ, Abdalla FC, Abeliovich H, Abraham RT, Acevedo-Arozena A, Adeli K, Agholme L, Agnello M, Agostinis P, Aguirre-Ghiso JA, et al: Guidelines for the use and interpretation of assays for monitoring autophagy. Autophagy 8: 445-544, 2012.

24. Mizushima N, Yoshimori T and Levine B: Methods in mammalian autophagy research. Cell 140: 313-326, 2010.

25. Matsunaga K, Saitoh T, Tabata K, Omori H, Satoh T, Kurotori N, Maejima I, Shirahama-Noda K, Ichimura T, Isobe T, et al: Two Beclin 1-binding proteins, Atg14L and Rubicon, reciprocally regulate autophagy at different stages. Nat Cell Biol 11: 385-396, 2009

26. Itakura $\mathrm{E}$, Kishi $\mathrm{C}$, Inoue $\mathrm{K}$ and Mizushima N: Beclin 1 forms two distinct phosphatidylinositol 3-kinase complexes with mammalian Atg14 and UVRAG. Mol Biol Cell 19: 5360-5372, 2008

27. Alm JJ, Heino TJ, Hentunen TA, Väänänen HK and Aro HT: Transient $100 \mathrm{nM}$ dexamethasone treatment reduces inter- and intraindividual variations in osteoblastic differentiation of bone marrow-derived human mesenchymal stem cells. Tissue Eng Part C Methods 18: 658-666, 2012.

28. Dalle Carbonare L, Arlot ME, Chavassieux PM, Roux JP, Portero NR and Meunier PJ: Comparison of trabecular bone microarchitecture and remodeling in glucocorticoid-induced and postmenopausal osteoporosis. J Bone Miner Res 16: 97-103, 2001.

29. Mazziotti G, Angeli A, Bilezikian JP, Canalis E and Giustina A: Glucocorticoid-induced osteoporosis: an update. Trends Endocrinol Metab 17: 144-149, 2006.

30. Canalis E, Bilezikian JP, Angeli A and Giustina A: Perspectives on glucocorticoid-induced osteoporosis. Bone 34: 593-598, 2004

31. Weinstein RS: Glucocorticoid-induced osteoporosis. Rev Endocr Metab Disord 2: 65-73, 2001

32. Weinstein RS, Jilka RL, Parfitt AM and Manolagas SC: Inhibition of osteoblastogenesis and promotion of apoptosis of osteoblasts and osteocytes by glucocorticoids. Potential mechanisms of their deleterious effects on bone. J Clin Invest 102: 274-282, 1998.

33. Chipuk JE, McStay GP, Bharti A, Kuwana T, Clarke CJ, Siskind LJ, Obeid LM and Green DR: Sphingolipid metabolism cooperates with BAK and BAX to promote the mitochondrial pathway of apoptosis. Cell 148: 988-1000, 2012.

34. Jilka RL, O'Brien CA, Roberson PK, Bonewald LF, Weinstein RS and Manolagas SC: Dysapoptosis of osteoblasts and osteocytes increases cancellous bone formation but exaggerates cortical porosity with age. J Bone Miner Res 29: 103-117, 2014.

35. Zhang T, Li Y, Park KA, Byun HS, Won M, Jeon J, Lee Y, Seok JH, Choi SW, Lee SH, et al: Cucurbitacin induces autophagy through mitochondrial ROS production which counteracts to limit caspase-dependent apoptosis. Autophagy 8: 559-576, 2012.

36. Maiuri MC, Zalckvar E, Kimchi A and Kroemer G: Self-eating and self-killing: crosstalk between autophagy and apoptosis. Nat Rev Mol Cell Biol 8: 741-752, 2007.

37. Rangaraju S, Verrier JD, Madorsky I, Nicks J, Dunn WA Jr and Notterpek L: Rapamycin activates autophagy and improves myelination in explant cultures from neuropathic mice. J Neurosci 30: 11388-11397, 2010.

38. Jiang M, Wei Q, Dong G, Komatsu M, Su Y and Dong Z: Autophagy in proximal tubules protects against acute kidney injury. Kidney Int 82: 1271-1283, 2012.

39. Warr MR, Binnewies M, Flach J, Reynaud D, Garg T, Malhotra R, Debnath J and Passegué E: FOXO3A directs a protective autophagy program in haematopoietic stem cells. Nature 494: 323-327, 2013.

40. Jung HS, Chung KW, Won Kim J, Kim J, Komatsu M, Tanaka K, Nguyen YH, Kang TM, Yoon KH, Kim JW, et al: Loss of autophagy diminishes pancreatic beta cell mass and function with resultant hyperglycemia. Cell Metab 8: 318-324, 2008.

41. Jia J, Yao W, Guan M, Dai W, Shahnazari M, Kar R, Bonewald L, Jiang JX and Lane NE: Glucocorticoid dose determines osteocyte cell fate. FASEB J 25: 3366-3376, 2011.

42. Xia HF, Jin XH, Cao ZF, Shi T and Ma X: miR-98 is involved in rat embryo implantation by targeting Bcl-xl. FEBS Lett 588: 574-583, 2014.

This work is licensed under a Creative Commons Attribution-NonCommercial-NoDerivatives 4.0 International (CC BY-NC-ND 4.0) License. 